\title{
Dynamic behaviors of a single species stage structure system with feedback control
}

\author{
Liyun Lai, Xiangqin Yu, Fengde Chen* \\ College of Mathematics and Computer Science, Fuzhou University, Fuzhou, Fujian, 350116, P. R. China.
}

\begin{abstract}
A single species stage structure system with feedback control is proposed and studied in this paper. Local and global stability property of the boundary equilibrium and the positive equilibrium are investigated, respectively. If the original system is globally stable, then we show that the feedback control only change the position of the unique positive equilibrium and retain the stable property. If the original system is extinct, then we show that the system with feedback control is also extinct. Some examples are presented to verify our main results.
\end{abstract}

Keywords: Stage structure, species, local stability, Lyapunov function, global stability.

2010 MSC: 34C25, 92D25.

(C)2019 All rights reserved.

\section{Introduction}

The aim of this paper is to investigate the dynamic behaviors of the following single species stage structure system with feedback control:

$$
\frac{d x_{1}}{d t}=\alpha x_{2}-\beta x_{1}-\delta_{1} x_{1}, \quad \frac{d x_{2}}{d t}=\beta x_{1}-\delta_{2} x_{2}-\gamma x_{2}^{2}-d x_{2} u, \quad \frac{d u}{d t}=-e u+f x_{2},
$$

where $\alpha, \beta, \delta_{1}, \delta_{2}, d, e, f$ and $\gamma$ are all positive constants, $x_{1}(t)$ and $x_{2}(t)$ are the densities of the immature and mature species at time $t, u$ is feedback control variable. The following assumptions are made in formulating the model (1.1).

1. The per capita birth rate of the immature population is $\alpha>0$, the per capita death rate of the immature population is $\delta_{1}>0$, the per capita death rate of the mature populations is proportional to the current mature populations with a proportionality constant $\delta_{2}>0, \beta>0$ denotes the surviving rate of immaturity to reach maturity, and the mature species is density dependent with the parameter $\gamma>0$.

2. The bilinear feedback mechanism $\left(d x_{2} u\right)$ is used to control the system.

\footnotetext{
${ }^{*}$ Corresponding author

Email addresses: 1310335147@qq.com (Liyun Lai), 1615657167@qq.com (Xiangqin Yu), fdchen@fzu.edu.cn (Fengde Chen)
}

doi: $10.22436 / \mathrm{mns} .05 .01 .01$

Received: 2019-01-25 Revised: 2019-05-08 Accepted: 2019-05-11 
During the last decades, many scholars investigated the dynamic behaviors of the stage structured species, see [1-44] and the references cited therein. Many new characters about the stage structured system were found, for example, Ma et al. [31] proposed and studied the persistent property of the following stagestructure predator-prey model

$$
\begin{aligned}
& \frac{d x_{1}(t)}{d t}=r_{1}(t) x_{2}(t)-d_{11} x_{1}(t)-r_{1}\left(t-\tau_{1}\right) e^{-d_{11} \tau_{1}} x_{2}\left(t-\tau_{1}\right), \\
& \frac{d x_{2}(t)}{d t}=r_{1}\left(t-\tau_{1}\right) e^{-d_{11} \tau_{1}} x_{2}\left(t-\tau_{1}\right)-d_{12} x_{2}(t)-b_{1}(t) x_{2}^{2}(t)-c_{1}(t) x_{2}(t) y_{2}(t), \\
& \frac{d y_{1}(t)}{d t}=r_{2}(t) y_{2}(t)-d_{22} y_{1}(t)-r_{2}\left(t-\tau_{2}\right) e^{-d_{22} \tau_{2}} y_{2}\left(t-\tau_{2}\right), \\
& \frac{d y_{2}(t)}{d t}=r_{2}\left(t-\tau_{2}\right) e^{-d_{22} \tau_{2}} y_{2}\left(t-\tau_{2}\right)-d_{21} y_{2}(t)-b_{2}(t) y_{2}^{2}(t)+c_{2}(t) y_{2}(t) x_{2}(t),
\end{aligned}
$$

where $x_{1}(t)$ and $x_{2}(t)$ denote the densities of the immature and mature prey species at time $t$, respectively, $y_{1}(t)$ and $y_{2}(t)$ represent the immature and mature population densities of predator species at time $t$, respectively, Chen et al. [5], Chen et al. [11], and Chen et al. [9] further investigated the permanence, extinction and global stability of the above system. They found that in system (1.2), the extinction of the prey species does not follows the extinction of the predator species, they conjectured that maybe the reason is in above system, the predator species has other food resource. Some other authors (see $[5,9-11,13,22,24-26,28,31,34])$ also applied the similar idea to establish the predator-prey model, cooperative model, and competition model, and many interesting results were obtained. For example, it's well known that May type cooperative system admits a unique positive equilibrium, which is globally attractive, however, Chen et al. [10] showed that with the introducing of the stage structure, May type cooperative system may admits partial survival property, that is, despite the cooperative between the species, the species may still be driven to the extinction due to the stage structure.

Recently, Khajanchi and Banerjee [23] proposed the following stage structure predator-prey model with ratio dependent functional response

$$
\begin{aligned}
\frac{d x_{1}}{d t} & =\alpha x_{2}(t)-\beta x_{1}(t)-\delta_{1} x_{1}(t), \\
\frac{d x_{2}}{d t} & =\beta x_{1}(t)-\delta_{2} x_{2}(t)-\gamma x_{2}^{2}(t)-\frac{\eta(1-\theta) x_{2}(t) y(t)}{g(1-\theta) x_{2}(t)+h y(t)}, \\
\frac{d y}{d t} & =\frac{u \eta(1-\theta) x_{2}(t) y(t)}{g(1-\theta) x_{2}(t)+h y(t)}-\delta_{3} y(t) .
\end{aligned}
$$

By constructing suitable Lyapunov function, the authors obtained a set of sufficient conditions which ensure the uniform persistence and global asymptotic stability of the system. It bring to our attention that in system (1.3), if we did not consider the predator species, then system (1.3) reduces to the following single species stage structured system.

$$
\frac{d x_{1}}{d t}=\alpha x_{2}(t)-\beta x_{1}(t)-\delta_{1} x_{1}(t), \quad \frac{d x_{2}}{d t}=\beta x_{1}(t)-\delta_{2} x_{2}(t)-\gamma x_{2}^{2}(t) .
$$

Obviously, $\mathrm{O}(0,0)$ is the boundary equilibrium of the system (1.4). However, in system (1.3), with the singularity of the ratio-dependent functional response, $\mathrm{O}(0,0,0)$ is not the equilibrium of the system, and so, the authors of [23] did not consider the stability property of $\mathrm{O}(0,0,0)$. Already, in [40], Xiao and Lei showed that under some suitable assumption, the boundary equilibrium $\mathrm{O}(0,0)$ of system (1.4) is globally asymptotically stable.

On the other hand, with the aim of alter the system structurally so as to make the population stabilize at value lower than the original system. Gopalsamy and Weng [18] first time proposed the following single species feedback control ecosystem

$$
\frac{d n(t)}{d t}=r n(t)\left[1-\left(\frac{a_{1} n(t)+a_{2} n(t-\tau)}{K}-c u(t)\right)\right], \frac{d u(t)}{d t}=-a u(t)+b n(t-\tau),
$$


where $a_{1}, a_{2}, c, a, b$ and $\tau$ are all positive constants. Such kind of "feedback" control variable can be implemented by means of a biological control or some harvesting procedure. By constructing some suitable Lyapunov functional, they showed that under the assumption $a_{1}>a_{2}>0$ holds, the system admits a unique positive equilibrium. Also, in [5], under the assumption $a_{1}=0$, the authors investigated the stability property of the positive equilibrium. For the case $a_{1}=0$, Gong et al. [17] investigated the Hopf bifurcation of system (1.5). Recently, Li and He [29] also investigated the Hopf bifurcation of the following single-species food-limited system with feedback control

$$
\frac{d u(t)}{d t}=u(t)\left(\frac{r(K-u(t))}{K+a u(t)}-c v(t-\tau)\right), \quad \frac{d v(t)}{d t}=-d v(t)+b u(t-\tau) .
$$

There are also many scholars argued that the non-autonomous case is more suitable. Chen et al. [12] studied the persistent property of the following single species feedback ecosystem

$$
\begin{aligned}
\frac{d N(t)}{d t} & =r(t) N(t)\left[1-\frac{N^{2}\left(t-\tau_{1}(t)\right)}{k^{2}(t)}-c(t) u\left(t-\tau_{2}(t)\right)\right], \\
\frac{d u(t)}{d t} & =-a(t) u(t)+b(t) N\left(t-\tau_{3}(t)\right) .
\end{aligned}
$$

In [12], by developing a new differential inequality, they showed that the system is always permanent. Based on the famous single species model proposed by Aiello and Freedman, Ding and Cheng [14] proposed the following single species stage-structured model with feedback control:

$$
\begin{aligned}
& \frac{d x_{1}(t)}{d t}=\alpha x_{2}(t)-\gamma x_{1}(t)-\alpha e^{-\gamma \tau} x_{2}(t-\tau), \\
& \frac{d x_{2}(t)}{d t}=\alpha e^{-\gamma \tau} x_{2}(t-\tau)-\beta x_{2}^{2}(t)-c x_{2}(t) u(t), \\
& \frac{d u(t)}{d t}=-a u(t)+b x_{2}(t) .
\end{aligned}
$$

In [14], it was shown that if the inequality

$$
a \beta>b c
$$

holds, then $x_{1}(t) \rightarrow x_{1}^{*}, x_{2}(t) \rightarrow x_{2}^{*}, u(t) \rightarrow u^{*}$ as $t \rightarrow+\infty$. This result is then generalized by Han et al. [19] to the infinite delay case. Noting that without the feedback control variable, the system (1.6) is degenerate to the following single species stage-structured system

$$
\frac{d x_{1}(t)}{d t}=\alpha x_{2}(t)-\gamma x_{1}(t)-\alpha e^{-\gamma \tau} x_{2}(t-\tau), \quad \frac{d x_{2}(t)}{d t}=\alpha e^{-\gamma \tau} x_{2}(t-\tau)-\beta x_{2}^{2}(t) .
$$

Already, Aiello and Freedman [1] had showed that system (1.8) admits a unique positive equilibrium, which is globally asymptotically stable. Hence, the result of Ding and Cheng [14] implies that under the assumption (1.7), the feedback control variable could only change the position of the equilibrium and did not change the stability property of the equilibrium. Recently, Li et al. [27] considered an autonomous Lotka-Volterra competitive system with infinite delays and feedback controls

$$
\begin{aligned}
& \frac{d x_{1}(t)}{d t}=x_{1}(t)\left(b_{1}-a_{11} x_{1}(t)-a_{12} \int_{0}^{+\infty} K_{1}(s) x_{2}(t-s) d s-c_{1} u_{1}(t)\right), \\
& \frac{d x_{2}(t)}{d t}=x_{2}(t)\left(b_{2}-a_{21} \int_{0}^{+\infty} K_{2}(s) x_{1}(t-s) d s-a_{22} x_{2}(t)-c_{2} u_{2}(t)\right), \\
& \frac{d u_{1}(t)}{d t}=-e_{1} u_{1}(t)+d_{1} x_{1}(t), \\
& \frac{d u_{2}(t)}{d t}=-e_{2} u_{2}(t)+d_{2} x_{2}(t),
\end{aligned}
$$


where $b_{i}, a_{i j}, e_{i}, d_{i}(i, j=1,2)$ are positive constants, $x_{i}(t)$ denotes the density of the population, $u_{i}(t)$ denotes the feedback control variable. Their study showed that the feedback controls only change the position of the unique positive equilibrium if the Lotka-Volterra competitive system is globally stable. However, for the extinct case, by choosing suitable control variables, extinct species can become globally stable, or still keep the property of extinction. Their findings showed that for the extinction case, feedback control variables may change the stability property of the system.

Since dynamic behaviors of system (1.4) is very different to that of the system (1.8), it's natural to ask: is it possible to avoid the extinction of the species in system (1.4) by choosing some suitable feedback control variable, if possible, whether the system could exhibit some similar dynamic behaviors as that of system (1.9)? The aim of this paper is to give a thoroughly analysis of the dynamic behaviors of system (1.1) and to answer the above problem. For more works on feedback control ecosystem, one could refer to [1-44] and the references cited therein.

The paper is arranged as follows. We will investigated the local stability property of the equilibria of system (1.1) in Section 2. In Section 3, By constructing some suitable Lyapunov function, we are able to investigate the global stability property of the equilibria. Section 4 presents some numerical simulations to show the feasibility of the main results. We end this paper by a briefly discussion.

\section{Local stability of the equilibria of system (1.1)}

The system (1.1) always admits the boundary equilibrium $\mathrm{O}_{1}(0,0,0)$. If $\beta \alpha>\delta_{2}\left(\beta+\delta_{1}\right)$ holds, then the system (1.1) admits a unique positive equilibrium $A_{1}\left(x_{1 *}, x_{2 *}, u_{*}\right)$, where

$$
x_{1 *}=\frac{\alpha x_{2}^{*}}{\beta+\delta_{1}}, \quad x_{2 *}=\frac{e\left(\alpha \beta-\delta_{2}\left(\beta+\delta_{1}\right)\right)}{(d f+e \gamma)\left(\beta+\delta_{1}\right)}=\frac{\frac{\alpha \beta}{\beta+\delta_{1}}-\delta_{2}}{\gamma+d \frac{f}{e}}, \quad u_{*}=\frac{f}{e} x_{2 *} .
$$

Obviously, $x_{1 *}, x_{2 *}$, and $u_{*}$ satisfy the equation

$$
\left\{\begin{array}{l}
\alpha x_{2 *}-\beta x_{1 *}-\delta_{1} x_{1 *}=0 \\
\beta x_{1 *}-\delta_{2} x_{2 *}-\gamma x_{2 *}^{2}-d x_{2 *} u_{*}=0 \\
-e u_{*}+f x_{2 *}=0
\end{array}\right.
$$

We shall now investigate the local stability property of the above equilibria.

The variational matrix of the system (1.1) is

$$
J\left(x_{1}, x_{2}, u\right)=\left(\begin{array}{ccc}
-\beta-\delta_{1} & \alpha & 0 \\
\beta & -\delta_{2}-2 \gamma x_{2}-d u & -d x_{2} \\
0 & f & -e
\end{array}\right) .
$$

Theorem 2.1. Assume that

$$
\alpha \beta<\delta_{2}\left(\beta+\delta_{1}\right)
$$

holds, then $\mathrm{O}_{1}(0,0,0)$ is locally asymptotically stable.

Proof. From (2.3) we could see that the Jacobian matrix of the system about the equilibrium point $\mathrm{O}_{1}(0,0,0)$ is given by

$$
\left(\begin{array}{ccc}
-\beta-\delta_{1} & \alpha & 0 \\
\beta & -\delta_{2} & 0 \\
0 & f & -e
\end{array}\right)
$$

The characteristic equation of above matrix is

$$
(\lambda+e)\left(\lambda^{2}+\left(\beta+\delta_{1}+\delta_{2}\right) \lambda+\beta \delta_{2}+\delta_{1} \delta_{2}-\alpha \beta\right)=0 .
$$

Hence, it has one negative characteristic root $\lambda_{1}=-e<0$, the other two characteristic roots are deter- 
mined by the equation

$$
\lambda^{2}+\left(\beta+\delta_{1}+\delta_{2}\right) \lambda+\beta \delta_{2}+\delta_{1} \delta_{2}-\alpha \beta=0
$$

Noting that under the assumption (2.4), the two characteristic roots of equation (2.6) satisfy

$$
\lambda_{2}+\lambda_{3}=-\left(\beta+\delta_{1}+\delta_{2}\right)<0, \quad \lambda_{2} \lambda_{3}=\beta \delta_{2}+\delta_{1} \delta_{2}-\alpha \beta>0,
$$

hence, $\lambda_{2}<0, \lambda_{3}<0$. Above analysis shows that the three characteristic roots of the matrix (2.5) are all negative, hence, $\mathrm{O}_{1}(0,0,0)$ is locally stable. This ends the proof of Theorem 2.1.

Theorem 2.2. Assume that

$$
\alpha \beta>\delta_{2}\left(\beta+\delta_{1}\right)
$$

holds, then $\mathrm{A}_{1}\left(\mathrm{x}_{1 *}, \mathrm{x}_{2 *}, \mathrm{u}_{*}\right)$ is locally asymptotically stable.

Proof. From (2.3) we could see that the Jacobian matrix of the system about the equilibrium point $A_{1}\left(x_{1 *}, x_{2 *}, u_{*}\right)$ is given by

$$
\left(\begin{array}{ccc}
-\beta-\delta_{1} & \alpha & 0 \\
\beta & -\delta_{2}-2 \gamma \chi_{2 *}-d u_{*} & -d x_{2 *} \\
0 & f & -e
\end{array}\right)
$$

The characteristic equation of system (1.1) at $A_{1}\left(x_{1 *}, x_{2 *}, u_{*}\right)$ is

$$
\lambda^{3}+\mathrm{B}_{1} \lambda^{2}+\mathrm{B}_{2} \lambda+\mathrm{B}_{3}=0,
$$

where

$$
\begin{aligned}
& B_{1}=d u_{*}+2 \gamma x_{2 *}+\beta+\delta_{1}+\delta_{2}+e, \\
& B_{2}=\left(\beta+\delta_{1}\right)\left(d u_{*}+2 \gamma x_{2 *}+\delta_{2}\right)-\alpha \beta+d x_{2 *} f+\left(d u_{*}+2 \gamma x_{2 *}+\beta+\delta_{1}+\delta_{2}\right) e, \\
& B_{3}=\left(\beta+\delta_{1}\right)\left(d u_{*}+2 \gamma x_{2 *}+\delta_{2}\right) e-\alpha \beta e+f d x_{2 *}\left(\beta+\delta_{1}\right) .
\end{aligned}
$$

From (2.1) and (2.2), we have

$$
d u_{*}+2 \gamma x_{2 *}+\delta_{2}=\beta \frac{x_{1 *}}{x_{2 *}}+\gamma x_{2 *}=\frac{\alpha \beta}{\beta+\delta_{1}}+\gamma x_{2 *} .
$$

Therefore,

$$
\begin{aligned}
\mathrm{B}_{1} & =d u_{*}+2 \gamma x_{2 *}+\beta+\delta_{1}+\delta_{2}+e>0, \\
B_{2} & =\left(\beta+\delta_{1}\right)\left(\frac{\alpha \beta}{\beta+\delta_{1}}+\gamma x_{2 *}\right)-\alpha \beta+d x_{2 *} f+\left(d u_{*}+2 \gamma x_{2 *}+\beta+\delta_{1}+\delta_{2}\right) e \\
& \geqslant d x_{2 *} f+\left(d u_{*}+2 \gamma x_{2 *}+\beta+\delta_{1}+\delta_{2}\right) e>0, \\
B_{3} & =\left(\beta+\delta_{1}\right)\left(d u_{*}+2 \gamma x_{2 *}+\delta_{2}\right) e-\alpha \beta e+f d x_{2 *}\left(\beta+\delta_{1}\right) \\
& =\left(\beta+\delta_{1}\right)\left(\frac{\alpha \beta}{\beta+\delta_{1}}+\gamma x_{2 *}\right) e-\alpha \beta e+f d x_{2 *}\left(\beta+\delta_{1}\right) \geqslant f d x_{2 *}\left(\beta+\delta_{1}\right)>0 .
\end{aligned}
$$

Set $K=d u_{*}+2 \gamma x_{2 *}+\delta_{2}$, then

$$
\begin{aligned}
\mathrm{B}_{1} \mathrm{~B}_{2}-\mathrm{B}_{3}= & \left(\mathrm{K}+\delta_{1}+\beta+e\right)\left[\left(\beta+\delta_{1}\right) \mathrm{K}+\left(\mathrm{K}+\beta+\delta_{1}\right) e\right. \\
& \left.-\alpha \beta+f \mathrm{fx}_{2 *}\right]-\left(\beta+\delta_{1}\right) \mathrm{Ke}+\alpha \beta e-f d x_{2 *}\left(\beta+\delta_{1}\right) \\
\geqslant & \left(\mathrm{K}+\delta_{1}+\beta+e\right)\left[\left(\beta+\delta_{1}\right) \frac{\alpha \beta}{\beta+\delta_{1}}+\left(\mathrm{K}+\beta+\delta_{1}\right) e\right.
\end{aligned}
$$




$$
\begin{aligned}
& \left.-\alpha \beta+f d x_{2 *}\right]-\left(\beta+\delta_{1}\right) K e+\alpha \beta e-f d x_{2 *}\left(\beta+\delta_{1}\right) \\
\geqslant & \left(K+\delta_{1}+\beta+e\right)\left[\left(K+\beta+\delta_{1}\right) e+f d x_{2 *}\right]-\left(\beta+\delta_{1}\right) K e+\alpha \beta e-f d x_{2 *}\left(\beta+\delta_{1}\right) \\
\geqslant & \left(K+\delta_{1}+\beta+e\right)\left(K+\beta+\delta_{1}\right) e-\left(\beta+\delta_{1}\right) K e+\alpha \beta e \\
\geqslant & \alpha \beta e>0 .
\end{aligned}
$$

By Hurwitz criterion, the three characteristic roots of the matrix (2.8) are all negative, hence, $A_{1}\left(x_{1 *}, x_{2 *}, u_{*}\right)$ is locally asymptotically stable. This ends the proof of Theorem 2.2.

\section{Global attractivity}

This section try to obtain some sufficient conditions which could ensure the global asymptotical stability of the equilibria of system (1.1).

Theorem 3.1. Assume that

$$
\alpha \beta<\delta_{2}\left(\beta+\delta_{1}\right)
$$

holds, then $\mathrm{O}_{1}(0,0,0)$ is globally asymptotically stable.

Proof. Condition (3.1) is equal to

$$
\frac{\alpha \beta}{\beta+\delta_{1}}-\delta_{2}+\frac{1}{2 f}\left(\delta_{2}-\frac{\alpha \beta}{\beta+\delta_{1}}\right) f<0
$$

We will prove Theorem 3.1 by constructing some suitable Lyapunov function. Let's define a Lyapunov function

$$
v_{1}\left(x_{1}, x_{2}, u\right)=\frac{\beta}{\beta+\delta_{1}} x_{1}+x_{2}+k_{3} u
$$

where

$$
k_{3}=\frac{1}{2 f}\left(\delta_{2}-\frac{\alpha \beta}{\beta+\delta_{1}}\right)
$$

One could easily see that the function $V_{1}$ is zero at the equilibrium $O(0,0,0)$ and is positive for all other positive values of $x_{1}, x_{2}$, and $u$. The time derivative of $v_{1}$ along the trajectories of (1.1) is

$$
\begin{aligned}
D^{+} v_{1}(t) & =\frac{\beta}{\beta+\delta_{1}}\left(\alpha x_{2}-\beta x_{1}-\delta x_{1}\right)+\beta x_{1}-\delta_{2} x_{2}-\gamma x_{2}^{2}-d u x_{2}-k_{3} e u+k_{3} f x_{2} \\
& =\left(\frac{\alpha \beta}{\beta+\delta_{1}}-\delta_{2}+k_{3} f\right) x_{2}-\gamma x_{2}^{2}-d u x_{2}-k_{3} e u .
\end{aligned}
$$

It then follows from (3.2) that $\mathrm{D}^{+} \mathrm{v}_{1}(\mathrm{t})<0$ strictly for all $x_{1}, x_{2}, \mathrm{u}>0$ except the boundary equilibrium $\mathrm{O}_{1}(0,0,0)$, where $\mathrm{D}^{+} \mathrm{V}_{1}(\mathrm{t})=0$. Thus, $\mathrm{V}_{1}\left(\mathrm{x}_{1}, \mathrm{x}_{2}, \mathrm{u}\right)$ satisfies Lyapunov's asymptotic stability theorem, and the boundary equilibrium $\mathrm{O}_{1}(0,0,0)$ of system $(1.1)$ is globally asymptotically stable. This completes the proof of Theorem 3.1.

Theorem 3.2. Assume that

$$
\alpha \beta>\delta_{2}\left(\beta+\delta_{1}\right)
$$

holds, then $\mathrm{A}_{1}\left(\mathrm{x}_{1 *}, \mathrm{x}_{2 *}, \mathrm{u}_{*}\right)$ is globally asymptotically stable.

Proof. We will prove Theorem 3.2 by constructing some suitable Lyapunov function. Let's define a Lyapunov function

$$
v_{2}\left(x_{1}, x_{2}, u\right)=k_{1}\left(x_{1}-x_{1 *}-x_{1 *} \ln \frac{x_{1}}{x_{1 *}}\right)+k_{2}\left(x_{2}-x_{2 *}-x_{2 *} \ln \frac{x_{2}}{x_{2 *}}\right)+k_{3}\left(u-u_{*}\right)^{2},
$$

where $k_{1}, k_{2}, k_{3}$ are some positive constants determined later. One could easily see that the function $v_{2}$ 
is zero at the equilibrium $A_{1}\left(x_{1 *}, x_{2 *}, u_{*}\right)$ and is positive for all other positive values of $x_{1}, x_{2}$ and $u$. The time derivative of $V_{2}$ along the trajectories of (1.1) is

$$
\begin{aligned}
\mathrm{D}^{+} \mathrm{v}_{2}(\mathrm{t})= & \mathrm{k}_{1} \frac{\mathrm{x}_{1}-\mathrm{x}_{1 *}}{\mathrm{x}_{1}} \dot{\mathrm{x}}_{1}+\mathrm{k}_{2} \frac{\mathrm{x}_{2}-\mathrm{x}_{2 *}}{\mathrm{x}_{2}} \dot{x}_{2} \\
= & \mathrm{k}_{1} \frac{\mathrm{x}_{1}-\mathrm{x}_{1 *}}{\mathrm{x}_{1}}\left(\alpha \mathrm{x}_{2}-\left(\beta+\delta_{1}\right) \mathrm{x}_{1}\right) \\
& +\mathrm{k}_{2} \frac{\mathrm{x}_{2}-\mathrm{x}_{2 *}}{\mathrm{x}_{2}}\left(\beta \mathrm{x}_{1}-\delta_{2} \mathrm{x}_{2}-\gamma \mathrm{x}_{2}^{2}-\mathrm{dux_{2 }}\right)+2 \mathrm{k}_{3}\left(\mathrm{u}-\mathrm{u}_{*}\right)\left(-\mathrm{eu}+\mathrm{f} \mathrm{x}_{2}\right) .
\end{aligned}
$$

Noting that from the relationship of $x_{1 *}, x_{2 *}$ and $u_{*}$ (see (2.2)), we have

$$
\begin{aligned}
\alpha x_{2}-\left(\beta+\delta_{1}\right) x_{1} & =\frac{\alpha}{x_{1 *}}\left(x_{2} x_{1 *}-x_{1} x_{2 *}\right)+\alpha x_{1} \frac{x_{2 *}}{x_{1 *}}-\left(\beta+\delta_{1}\right) x_{1} \\
& =\frac{\alpha}{x_{1 *}}\left(-x_{2}\left(x_{1}-x_{1 *}\right)+x_{1}\left(x_{2}-x_{2 *}\right)\right)
\end{aligned}
$$

also, from (2.1) and (2.3), we have

$$
\begin{aligned}
\beta x_{1}-\delta_{2} x_{2}-\gamma x_{2}^{2}-d u x_{2} & =\frac{\beta}{x_{2 *}}\left(x_{1} x_{2 *}-x_{2} x_{1 *}\right)+\beta x_{2} \frac{x_{1 *}}{x_{2 *}}-\delta_{2} x_{2}-\gamma x_{2}^{2}-d u x_{2} \\
& =\frac{\beta}{x_{2 *}}\left(x_{1} x_{2 *}-x_{1} x_{2}+x_{1} x_{2}-x_{2} x_{1 *}\right)+\left(\frac{\alpha \beta}{\beta+\delta_{1}}-\delta_{2}\right) x_{2}-\gamma x_{2}^{2}-d u x_{2} \\
& =\frac{\beta}{x_{2 *}}\left(x_{1} x_{2 *}-x_{1} x_{2}+x_{1} x_{2}-x_{2} x_{1 *}\right)+\left(\gamma+d \frac{f}{e}\right) x_{2 *} x_{2}-\gamma x_{2}^{2}-d u x_{2} \\
& =\frac{\beta}{x_{2 *}}\left(x_{1}\left(x_{2 *}-x_{2}\right)+x_{2}\left(x_{1}-x_{1 *}\right)\right)+\gamma x_{2}\left(x_{2 *}-x_{2}\right)+d x_{2}\left(u_{*}-u\right),
\end{aligned}
$$

from the third equation of (2.2), we have

$$
-e u+f x_{2}=-e u+f x_{2}+e u_{*}-f x_{2 *}=-e\left(u-u_{*}\right)+f\left(x_{2}-x_{2 *}\right) .
$$

Applying (3.4)-(3.6) to (3.3) leads to

$$
\begin{aligned}
\mathrm{D}^{+} \mathrm{v}_{2}(\mathrm{t})= & k_{1} \frac{x_{1}-x_{1 *}}{x_{1}} \frac{\alpha}{x_{1 *}}\left(-x_{2}\left(x_{1}-x_{1 *}\right)+x_{1}\left(x_{2}-x_{2 *}\right)\right) \\
& +k_{2} \frac{x_{2}-x_{2 *}}{x_{2}} \frac{\beta}{x_{2 *}}\left(x_{1}\left(x_{2 *}-x_{2}\right)+x_{2}\left(x_{1}-x_{1 *}\right)\right) \\
& +k_{2} \gamma x_{2} \frac{x_{2}-x_{2 *}}{x_{2}}\left(x_{2 *}-x_{2}\right)+k_{2} d x_{2} \frac{x_{2}-x_{2 *}}{x_{2}}\left(u_{*}-u\right) \\
& +2 k_{3}\left(u-u_{*}\right)\left(-e\left(u-u_{*}\right)+f\left(x_{2}-x_{2 *}\right)\right) \\
= & -\frac{k_{1} \alpha x_{2}}{x_{1} x_{1 *}}\left(x_{1}-x_{1 *}\right)^{2}+\left(\frac{k_{1} \alpha}{x_{1 *}}+\frac{k_{2} \beta}{x_{2 *}}\right)\left(x_{1}-x_{1 *}\right)\left(x_{2}-x_{2 *}\right) \\
& -\frac{k_{2} \beta x_{1}}{x_{2} x_{2 *}}\left(x_{2}-x_{2 *}\right)^{2}-k_{2} \gamma\left(x_{2}-x_{2 *}\right)^{2}+k_{2} d\left(x_{2}-x_{2 *}\right)\left(u_{*}-u\right) \\
& -2 k_{3} e\left(u-u_{*}\right)^{2}+2 k_{3} f\left(u-u_{*}\right)\left(x_{2}-x_{2 *}\right)
\end{aligned}
$$

Now let's choose $k_{2}=1, k_{1}=\frac{\beta x_{1 *}}{x_{2 *},}, k_{3}=\frac{d}{2 f}$, then

$$
\begin{aligned}
\mathrm{D}^{+} \mathrm{v}_{2}(\mathrm{t})= & -\frac{\beta x_{2}}{x_{1} x_{2 *}}\left(x_{1}-x_{1 *}\right)^{2}+\frac{2 \beta}{x_{2 *}}\left(x_{1}-x_{1 *}\right)\left(x_{2}-x_{2 *}\right) \\
& -\frac{\beta x_{1}}{x_{2} x_{2 *}}\left(x_{2}-x_{2 *}\right)^{2}-\gamma\left(x_{2}-x_{2 *}\right)^{2}-\frac{d e}{f}\left(u-u_{*}\right)^{2} \\
= & -\frac{\beta}{x_{2 *}}\left[\sqrt{\frac{x_{2}}{x_{1}}}\left(x_{1}-x_{1 *}\right)-\sqrt{\frac{x_{1}}{x_{2}}}\left(x_{2}-x_{2 *}\right)\right]^{2}-\gamma\left(x_{2}-x_{2 *}\right)^{2}-\frac{d e}{f}\left(u-u_{*}\right)^{2}
\end{aligned}
$$


Hence, $D^{+} V_{2}(t)<0$ strictly for all $x_{1}, x_{2}, u>0$ except the positive equilibrium $A\left(x_{1 *}, x_{2 *}, u_{*}\right)$, where $\mathrm{D}^{+} \mathrm{V}_{2}(\mathrm{t})=0$. Thus, $\mathrm{V}_{2}\left(\mathrm{x}_{1}, \mathrm{x}_{2}, \mathrm{u}\right)$ satisfies Lyapunov's asymptotic stability theorem, and the positive equilibrium $A_{1}\left(x_{1 *}, x_{2 *}, u_{*}\right)$ of system (1.1) is globally asymptotically stable. This completes the proof of Theorem 3.2.

Remark 3.3. Compared with Theorems 3.1 and 3.2, we showed that if the system is globally stable, then the feedback control variable only change the position of the unique positive equilibrium and retain the stable property. If the system is extinct, then we show that the system with feedback control is also extinct.

Remark 3.4. For the case the system admits a unique positive equilibrium, from Theorems 3.2 and 2.2 and the expression of $x_{1 *}, x_{2 *}$ (see (2.1)), one could easily see that $x_{2 *}<x_{2}^{*}, x_{1 *}<x_{1}^{*}$, where $\left(x_{1}^{*}, x_{2}^{*}\right)$ is the unique positive equilibrium of the system (1.4). Hence, the feedback control variable alter the system structurally so as to make the population stabilize at value lower than the original system.

Remark 3.5. Condition $\beta \alpha>\delta_{2}\left(\beta+\delta_{1}\right)$ is sufficient and necessary to ensure the systems (1.1) and (1.4) admit a unique positive equilibrium, hence, we could draw the conclusion: if the system (1.4) admits the unique positive equilibrium, the feedback control variables will have no influence on the stability of the system.

\section{Numeric simulations}

Now let's consider the following examples.

Example 4.1. Let's consider the single species stage structure system with feedback control:

$$
\frac{d x_{1}}{d t}=3 x_{2}-x_{1}-x_{1}, \quad \frac{d x_{2}}{d t}=x_{1}-x_{2}-x_{2}^{2}-x_{2} u, \quad \frac{d u}{d t}=-u+x,
$$

here we choose $\beta=\delta_{1}=\delta_{2}=\gamma=e=f=1, \alpha=3$. Hence

$$
\alpha \beta=3>2=\delta_{2}\left(\beta+\delta_{1}\right) .
$$

It follows from Theorem 3.2 that the unique positive equilibrium $A_{1}\left(\frac{3}{8}, \frac{1}{4}, \frac{1}{4}\right)$ is globally asymptotically stable. Numeric simulations (Figs. 1, 2, 3) also support this assertion.

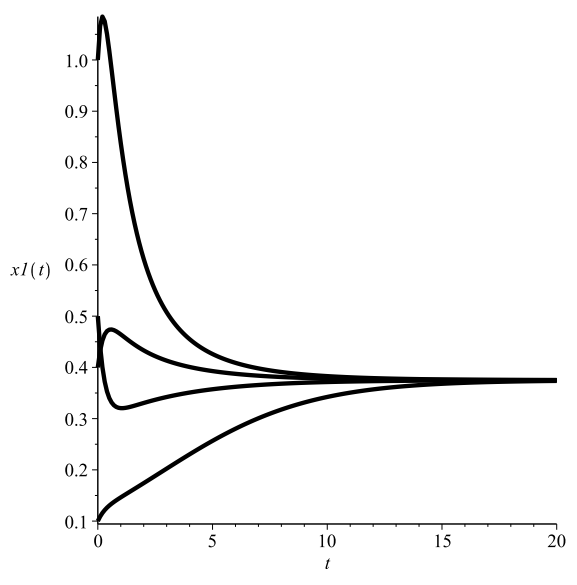

Figure 1: Dynamics behaviors of the first component $x_{1}(t)$ of system (4.1), here we take $\alpha=3$ and the initial conditions $\left(\mathrm{x}_{1}(0), \mathrm{x}_{2}(0), \mathrm{u}(0)\right)=$ $(0.5,0.1,0.2),(0.1,0.1,0.1),(1,1,1) \quad$ and $(0.4,0.4,0.4)$, respectively.

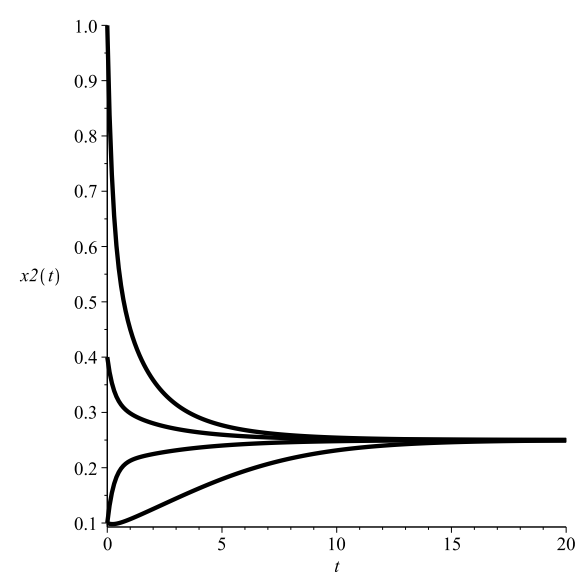

Figure 2: Dynamics behaviors of the second component $x_{2}(t)$ of the system (4.1). Here, we take $\alpha=3$ and the initial conditions $\left(x_{1}(0), x_{2}(0), u(0)\right)=$ $(0.5,0.1,0.2),(0.1,0.1,0.1),(1,1,1)$ and $(0.4,0.4,0.4)$, respectively. 


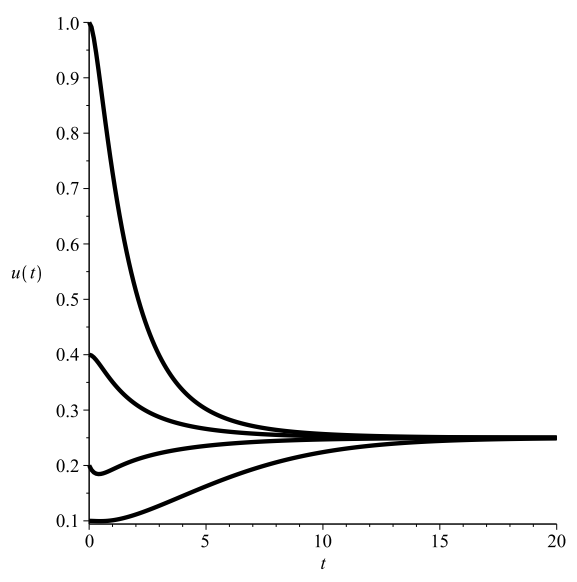

Figure 3: Dynamics behaviors of the third component $u(t)$ of the system (4.1), here we take $\alpha=$ 3 and the initial conditions $\left(x_{1}(0), x_{2}(0), u(0)\right)=$ $(0.5,0.1,0.2),(0.1,0.1,0.1),(1,1,1)$ and $(0.4,0.4,0.4)$, respectively.

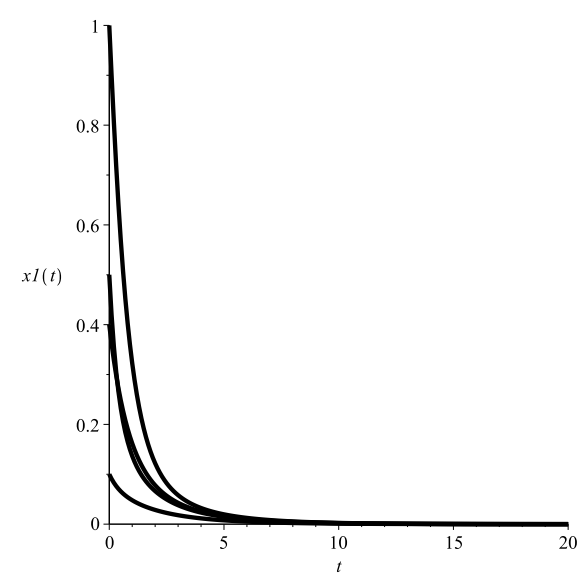

Figure 4: Dynamics behaviors of the first component $x_{1}(t)$ of the system (4.2), here we take $\alpha=$ 1 and the initial conditions $\left(\mathrm{x}_{1}(0), \mathrm{x}_{2}(0), \mathrm{u}(0)\right)=$ $(0.5,0.1,0.2),(0.1,0.1,0.1),(1,1,1)$ and $(0.4,0.4,0.4)$, respectively.

Example 4.2. Let's consider the single species stage structure system with feedback control:

$$
\frac{d x_{1}}{d t}=x_{2}-x_{1}-x_{1}, \quad \frac{d x_{2}}{d t}=x_{1}-x_{2}-x_{2}^{2}-x_{2} u, \quad \frac{d u}{d t}=-u+x,
$$

here we choose $\beta=\delta_{1}=\delta_{2}=\gamma=e=f=\alpha=1$. Hence

$$
\alpha \beta=1<2=\delta_{2}\left(\beta+\delta_{1}\right)
$$

It follows from Theorem 3.1 that the boundary equilibrium $\mathrm{O}_{1}(0,0,0)$ is globally asymptotically stable. Numeric simulations (Figs. 4, 5, 6) also support this assertion.

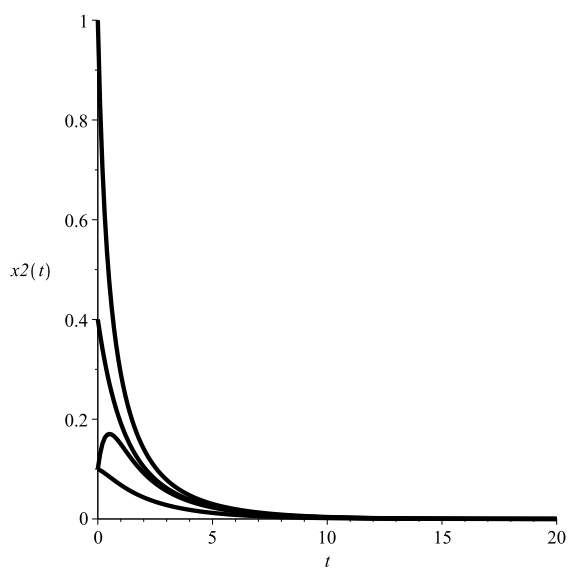

Figure 5: Dynamics behaviors of the second component $x_{2}(t)$ of the system (4.2), here we take $\alpha=$ 1 and the initial conditions $\left(x_{1}(0), x_{2}(0), u(0)\right)=$ $(0.5,0.1,0.2),(0.1,0.1,0.1),(1,1,1)$ and $(0.4,0.4,0.4)$, respectively.

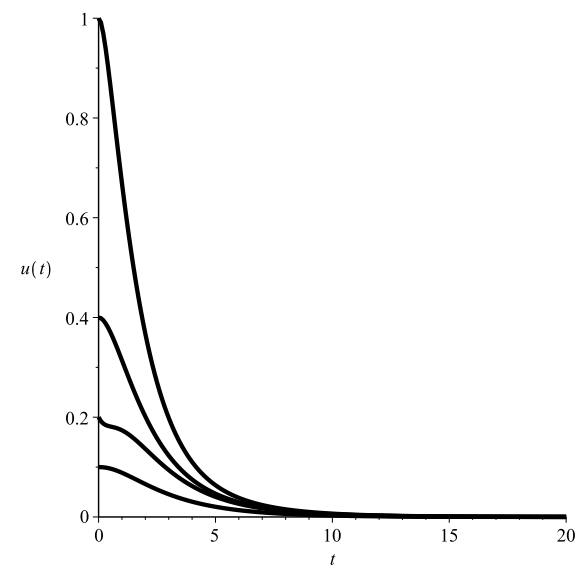

Figure 6: Dynamics behaviors of the third component $u(t)$ of the system (4.2), here we take $\alpha=$ 1 and the initial conditions $\left(x_{1}(0), x_{2}(0), u(0)\right)=$ $(0.5,0.1,0.2),(0.1,0.1,0.1),(1,1,1)$ and $(0.4,0.4,0.4)$, respectively. 


\section{Conclusion}

Since the pioneering work of Gopalsamy and Weng [18], the study of the dynamic behaviors of ecosystem with feedback control system becomes one of the main topics of mathematics biology (see [18-32] and the references cited therein). Among those works, two papers seems very interesting, the first one is Ding and Cheng [14], they proposed the single species stage structured system with feedback control (system (1.6)), though they showed the system admits unique positive equilibrium, they needed some additional condition (inequality (1.7)) to ensure the global asymptotically stable of the positive equilibrium. The second interesting paper is wrote by Li et al. [27], they proposed a competitive system with feedback controls, they found that for the extinct case, by choosing suitable control variables, extinct species can become globally stable, or still keep the property of extinction, which means that the feedback control mechanism is one of the essential method to avoid the extinction of the species. On the other hand, recently, Khajanchi and Banerjee [23] proposed a stage structured predator prey system (system (1.3)), and their success stimulated us to propose the system (1.1).

It seems that our results are also very interesting. We showed that if the system (1.4) admits a unique positive equilibrium, then feedback control variable could only alter the positive equilibrium to a lower case and could not change the stability property of the positive equilibrium, and for the extinct case, dislike the results of $\mathrm{Li}$ et al. [27], we show that the system still driven to extinction, and feedback mechanism could not avoid the extinct of the species.

Condition (2.7) could also be rewrite as $\alpha>\delta_{2}\left(1+\frac{\delta_{1}}{\beta}\right)$, from this, we could find that the birth rate of the immature species and death rate of the mature species play most important role on the persistent and stability of the system. Promoting the birth rate of the immature species and reducing the death rate of the mature species will increasing the stability of the system.

\section{Acknowledgment}

This work is supported by the National Natural Science Foundation of China under Grant (11601085) and the Natural Science Foundation of Fujian Province (2017J01400).

\section{References}

[1] W. G. Aiello, H. I. Freedman, A time-delay model of single-species growth with stage structure, Math. Biosci., 101 (1990), 139-144. 1, 1, 1

[2] L. Chen, F. D. Chen, Global stability of a Leslie-Gower predator-prey model with feedback controls, Appl. Math. Lett., 22 (2009), 1330-1334.

[3] L. J. Chen, F. D. Chen, Extinction in a discrete Lotka-Volterra competitive system with the effect of toxic substances and feedback controls, Int. J. Biomath., 8 (2015), 13 pages.

[4] L. J. Chen, L. J. Chen, Z. Li, Permanence of a delayed discrete mutualism model with feedback controls, Math. Comput. Modelling, 50 (2009), 1083-1089.

[5] F. D. Chen, W. L. Chen, Y. M. Wu, Z. Z. Ma, Permanece of a stage-structured predator-prey system, Appl. Math. Comput., 219 (2013), 8856-8862. 1, 1

[6] X. Y. Chen, C. L. Shi, Y. Q. Wang, Almost periodic solution of a discrete Nicholson's blowflies model with delay and feedback control, Adv. Difference Equ., 2016 (2016), 15 pages.

[7] L. J. Chen, J. T. Sun, Global stability of an SI epidemic model with feedback controls, Appl. Math. Lett., 28 (2014), 53-55.

[8] F. D. Chen, H. N. Wang, Dynamic behaviors of a Lotka-Volterra competitive system with infinite delay and single feedback control, J. Nonlinear Funct. Anal., 2016 (2016), 11 pages.

[9] F. D. Chen, H. N. Wang, Y. H. Lin, W. L. Chen, Global stability of a stage-structured predator-prey system, Appl. Math. Comput., 223 (2013), 45-53. 1

[10] F. D. Chen, X. D. Xie, X. F. Chen, Dynamic behaviors of a stage-structured cooperation model, Commun. Math. Biol. Neurosci., 2015 (2015), 19 pages. 1

[11] F. D. Chen, X. D. Xie, Z. Li, Partial survival and extinction of a delayed predator-prey model with stage structure, Appl. Math. Comput., 219 (2012), 4157-4162. 1

[12] F. D. Chen, J. H. Yang, L. J. Chen, Note on the persistent property of a feedback control system with delays, Nonlinear Anal. Real World Appl., 11 (2010), 1061-1066. 1 
[13] F. D. Chen, M. S. You, Permanence, extinction and periodic solution of the predator-prey system with BeddingtonDeAngelis functional response and stage structure for prey, Nonlinear Anal. Real World Appl., 9 (2008), $207-221$. 1

[14] X. Q. Ding, S. H. Cheng, The stability of a delayed stage-structured population growth model with feedback controls, J. Biomath., 21 (2006), 225-232. 1, 1, 1, 5

[15] Y.-H. Fan, L.-L. Wang, Permanence for a discrete model with feedback control and delay, Discrete Dyn. Nat. Soc., 2008 (2008), 8 pages.

[16] Y.-H. Fan, L.-L. Wang, Global asymptotical stability of a Logistic model with feedback control, Nonlinear Anal. Real World Appl., 11 (2010), 2686-2697.

[17] X. Gong, X. Xie, R. Han, L. Yang, Hopf bifurcation in a delayed logistic growth with feedback control, Commun. Math. Biol. Neurosci., 2015 (2015), 16 pages. 1

[18] K. Gopalsamy, P. X. Weng, Feedback regulation of Logistic growth, Internat. J. Math. Sci., 16 (1993), 177-192. 1, 5

[19] R. Y. Han, F. D. Chen, Global stability of a commensal symbiosis model with feedback controls, Commun. Math. Biol. Neurosci., 2015 (2015), 10 pages. 1

[20] R. Y. Han, F. D. Chen, X. D. Xie, Z. S. Miao, Global stability of May cooperative system with feedback controls, Adv. Difference Equ., 2015 (2015), 10 pages.

[21] R. Y. Han, X. D. Xie, F. D. Chen, Permanence and global attractivity of a discrete pollination mutualism in plant-pollinator system with feedback controls, Adv. Difference Equ., 2016 (2016), 17 pages.

[22] R. Y. Han, L. Y. Yang, Y. L. Xue, Global attractivity of a single species stage-structured model with feedback control and infinite delay, Commun. Math. Biol. Neurosci., 2015 (2015), 16 pages. 1

[23] S. Khajanchi, S. Banerjee, Role of constant prey refuge on stage structure predator-prey model with ratio dependent functional response, Appl. Math. Comput., 314 (2017), 193-198. 1, 1, 5

[24] Z. Li, F. D. Chen, Extinction in periodic competitive stage-structured Lotka-Volterra model with the effects of toxic substances, J. Comput. Appl. Math., 231 (2009), 143-153. 1

[25] T. T. Li, F. D. Chen, J. H. Chen, Q. X. Lin, Stability of a mutualism model in plant-pollinator system with stage-structure and the Beddington-DeAngelis functional response, J. Nonlinear Funct. Anal., 2017 (2017), 13 pages.

[26] Z. Li, M. Han, F. D. Chen, Global stability of stage-structured predator-prey model with modified Leslie-Gower and Holling-type II schemes, Int. J. Biomath., 6 (2012), 13 pages. 1

[27] Z. Li, M. Han, F. D. Chen, Influence of feedback controls on an autonomous Lotka-Volterra competitive system with infinite delays, Nonlinear Anal. Real World Appl., 14 (2013), 402-413. 1, 5

[28] Z. Li, M. Han, F. D. Chen, Global stability of a predator-prey system with stage structure and mutual interference, Discrete Contin. Dyn. Syst. Ser. B, 19 (2014), 173-187. 1

[29] Z. Li, M. X. He, Hopf bifurcation in a delayed food-limited model with feedback control, Nonlinear Dynam., 76 (2014), 1215-1224. 1

[30] Y. H. Lin, X. D. Xie, F. D. Chen, T. T. Li, Convergences of a stage-structured predator-prey model with modified LeslieGower and Holling-type II schemes, Adv. Difference Equ., 2016 (2016), 19 pages.

[31] Z.-H. Ma, Z.-Z. Li, S.-F. Wang, T. Li, F.-P. Zhang, Permanence of a predator-prey system with stage structure and time delay, Appl. Math. Comput., 201 (2008), 65-71. 1, 1

[32] Z. H. Ma, S. F. Wang, Permanence of a food-chain system with stage structure and time delay, Commun. Math. Biol. Neurosci., 2017 (2017), 8 pages. 5

[33] Z. S. Miao, F. D. Chen, J. M. Liu, L. Q. Pu, Dynamic behaviors of a discrete Lotka-Volterra competitive system with the effect of toxic substances and feedback controls, Adv. Difference Equ., 2017 (2017), 19 pages.

[34] L. Q. Pu, Z. S. Miao, R. Y. Han, Global stability of a stage-structured predator-prey model, Commun. Math. Biol. Neurosci., 2015 (2015), 13 pages. 1

[35] C. L. Shi, X. Y. Chen, Y. Q. Wang, Feedback control effect on the Lotka-Volterra prey-predator system with discrete delays, Adv. Difference Equ., 2017 (2017), 13 pages.

[36] C. L. Shi, Z. Li, F. D. Chen, Extinction in a nonautonomous Lotka-Volterra competitive system with infinite delay and feedback controls, Nonlinear Anal. Real World Appl., 13 (2012), 2214-2226.

[37] X. Y. Song, L. M. Cai, A. U. Neumann, Ratio-dependent predator-prey system with stage structure for prey, Discrete Contin. Dyn. Syst. Ser. B, 4 (2004), 747-758.

[38] Y. J. Wang, Periodic and almost periodic solutions of a nonlinear single species discrete model with feedback control, Appl. Math. Comput., 219 (2013), 5480-5486.

[39] H. L. Wu, F. D. Chen, Harvesting of a single-species system incorporating stage structure and toxicity, Discrete Dyn. Nat. Soc., 2009 (2009), 16 pages.

[40] A. Xiao, C. Q. Lei, Dynamic behaviors of a non-selective harvesting single species stage-structured system incorporating partial closure for the populations, Adv. Difference Equ., 2018 (2018), 16 pages. 1

[41] J. B. Xu, Z. D. Teng, Permanence for a nonautonomous discrete single-species system with delays and feedback control, Appl. Math. Lett., 23 (2010), 949-954.

[42] K. Yang, Z. S. Miao, F. D. Chen, X. D. Xie, Influence of single feedback control variable on an autonomous Holling II type cooperative system, J. Math. Anal. Appl., 435 (2016), 874-888.

[43] S. B. Yu, Extinction for a discrete competition system with feedback controls, Adv. Difference Equ., 2017 (2017), 10 pages. 
[44] T. W. Zhang, Y. K. Li, Y. Ye, Persistence and almost periodic solutions for a discrete fishing model with feedback control, Commun. Nonlinear Sci. Numer. Simul., 16 (2011), 1564-1573. 1, 1 